\title{
An Improved Particle Swarm Optimization(PSO)-Based MPPT Strategy for PV System
}

\author{
Tianmeng Wei ${ }^{1}$, Dongliang Liu ${ }^{1,2, a}$, and Chuanfeng Zhang ${ }^{1}$ \\ ${ }^{1}$ School of Automation Engineering, Hangzhou Dianzi University, Hangzhou, China \\ ${ }^{2}$ Wolong ELectric Group Co.,Ltd, Shangyu, China
}

\begin{abstract}
Under partially shaded conditions, the P-U curve of PV array contains multiple extreme points. General MPPT methods may misjudge the MPP and trap in the local extreme point, which will cause low working efficiency. Although the traditional PSO algorithm can accurately track the maximum power point under this condition, the optimizing process fluctuates obviously and the tracking speed can be improved. In order to solve these problems, an improved PSO algorithm is proposed. The initial positions of the particles are located by analysing the relationship of the I-U and P-U characteristic curves. It is more closed to the maximum power point. So the efficiency of PSO algorithm is improved. To evaluate the effectiveness of this method, the simulation model is established in MATLAB/Simulink. Under partially shaded conditions the algorithm can track the maximum power point quickly and accurately.
\end{abstract}

Keywords-Maximum power point Tracking(MPPT), partial shading, particle swarm optimization(PSO), photovoltaic $(P V)$ system.

\section{Introduction}

With the rapid increase in the environmental costs of fossil fuels, many countries are seeking to meet their energy needs through renewable energy sources, particularly solar energy. However, in the developing process of solar energy, a number of problems are eager to be solved. One of the problems, experts in the whole word focusing on, is that the efficiency of the optimization process of maximum power point tracking. In recent years, numerous MPPT methods are presented to track MPPs correctly and to improve the dynamic and steadystate tracking performance. Perturb and Observe $(\mathrm{P} \& \mathrm{O})$ is the most popular among these strategies. However, in the actual cases, due to the influence of partial shading, mismatch between components and ageing of solar panels, P-U characteristic of PV arrays appears multi extreme points. In this case, traditional method will trap in local maximum points and cause fail of optimization. To solve this problem, experts did a lot of researches. Reference [4] proposed an improved global scanning method. This method can track MPPT more accurately, but the optimization process required for a long time, which is easy to cause power loss. Reference [5] using Fibonacci MPP optimization method. Although this method can adapt for the changing condition, it convergence for a long time and lack of practicality. Reference [6] using traditional particle swarm optimization algorithm, it chooses the randomness position as initial value of particles. The optimization process is slow and easy to lose MPP.
As discussed before, a new and feasible MPPT method need to be developed. In this paper, an improved particle swarm optimization algorithm is proposed. Based on traditional particle swarm optimization algorithm, this method initializes the initial value of particles by analysing the derivative of PV arrays' I-U curve. It can make sure that the improved method will not trap in the local maximum power point and find the global maximum power point faster than before. That means the efficiency of PSO algorithm has been improved. In addition, different from the general particle swarm algorithm the fitness value of this improved algorithm is the real-time sampling of PV array's output power. It leaves out the trouble of repeated iteration and improves the operation efficiency; As same as the general PSO algorithm, the termination and restart conditions are set to avoid the vibration tending to the maximum power point. To evaluate the effectiveness of the proposed method, MATLAB simulations are carried out under the multi extreme point conditions. It is verified that the proposed method can quickly complete the MPP optimization process, improve the efficiency of the PV system and enhance the capacity of the system.

\section{Modeling of PV array}

In this paper, a $\{3 \times 3\}$ PV array as shown in Fig.1 is the PV array structure of simulation. To avoid the hot spot effect of PV products, each component is paralleling a bypass diode. At the same time for each series branch has a blocking diode in series, which can protect the operation of the PV array [11-12]. In the simulation, the component

\footnotetext{
${ }^{\mathrm{a}}$ Corresponding author: liudl@hdu.edu.cn
} 
parameters are set following the parameters of solar panel JLS60Pj(250). Under standard condition (Temperature is $25^{\circ} \mathrm{C}$, Sunlight is $1000 \mathrm{~W} / \mathrm{m} 2$ ) solar panels' open circuit voltage, short circuit current of PV panels, MPP's current value and the voltage value of the MPP are $36.5 \mathrm{~V}, 30.4 \mathrm{~V}$, 9.1A and 8.2A respectively.

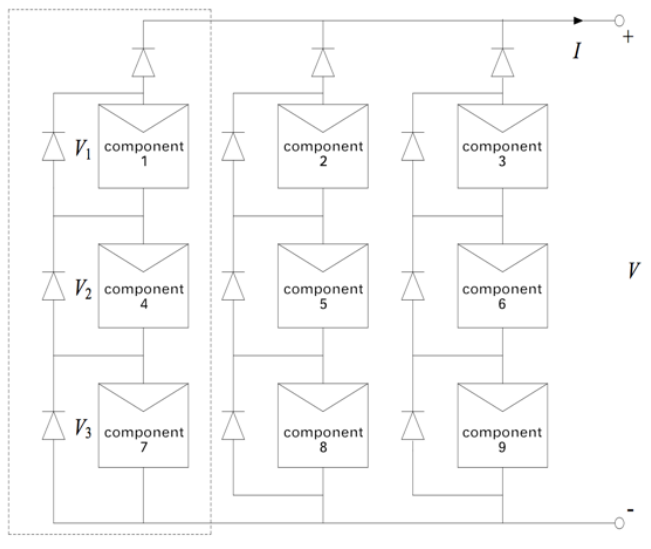

Fig.1 $\{3 \times 3\}$ Configuration of PV array

To facilitate the analysis and understanding, we choose the double peak situation in this paper. The first peak is local maximum power point, and the second peak is the global maximum power point. We set the parameters of components 1 to 6 as standard condition and the parameters of components 7 to 9 as $\mathrm{T}=25^{\circ} \mathrm{C}$, $\mathrm{S}=700 \mathrm{~W} / \mathrm{m} 2$. In order to get the PV array's output characteristic equation, it is necessary to analyse the internal physical structure of PV array. Because the three branches of PV array have the same characteristic, we choose the components 1, 4, and 7 (as shown in the dotted box in Fig.1) as the physical model to get the mathematical model of PV array. Fig. 2 shows the structure of circuit model.

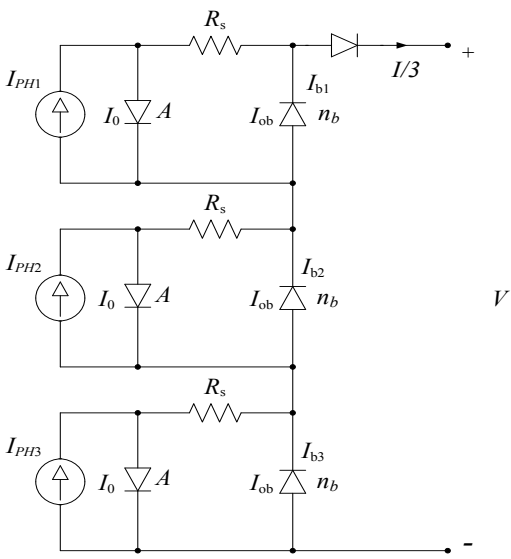

Fig.2 Circuit model of serial module with bypass diode

In this model, three pieces of photovoltaic cells' photo-generated current are $I_{P H 1} 、 I_{P H 2} 、 I_{P H 3}$ respectively. The current flowing through this branch is $I / 3 . R_{\mathrm{s}}$ is the resistance of the solar cell. $A 、 n_{\mathrm{b}}$ is the diode's impact factor. $I_{0} 、 I_{\mathrm{ob}}$ is the diode's reverse saturation current. T is absolute temperature $\mathrm{K}$ and $\mathrm{q}$ is electronic charge constant. Because component 1 and 4 are in the same temperature and radiation conditions, $I_{P H 1}$ is equates to
$I_{P H 2}$. According to the previous settings, the radiation condition of component 7 is less than component 1 and 4, so $\mathrm{I}_{\mathrm{PH} 1}>\mathrm{I}_{\mathrm{PH} 3}$. Commonly used simplified current equation of photovoltaic cell can be expressed as follow:

$$
I=I_{P H}-I_{0}\left[\exp \left(\frac{U+I R_{s}}{A k T}\right)-1\right]
$$

Because $I_{P H 1}$ is greater than $I_{P H 2}$, when the output current is very big the bypass diode of component 7 is in a conducting state. Then the excess current of component 1 and 4 will flow through this diode to the external. At this stage, only component 1 and 4 output power. As for component 7 , it is absorbing part of the power from component 1 and 4; When the output current of component 1 and 4 is equal to component 7's, the bypass diode of component 7 will in blocking state. Then all the three components output power. Based on the above analysis, under shading condition the solar cell's output characteristic equation is shown as follow[13]:

$$
U=\left\{\begin{array}{l}
2 \frac{A k T}{q} \ln \left(\frac{I_{P H 1}-I / 3}{I_{0}}+1\right)-2 I R_{S} / 3- \\
\frac{n_{b} k T_{b}}{q} \ln \left(\frac{I / 3-I_{P H 3}}{I_{o b}}+1\right), I_{P H 3}<I / 3<I_{P H 1} \\
2 \frac{A K T}{q} \ln \left(\frac{I_{P H 1}-I / 3}{I_{0}}+1\right)-I R_{s}+ \\
\frac{A k T}{q} \ln \left(\frac{I_{P H 3}-I / 3}{I_{0}}+1\right), 0<I / 3<I_{P H 3}
\end{array}\right.
$$

According to the circuit equation $\mathrm{P}=\mathrm{UI}$ and equation (2), the power output function is able to get, as shown in equation (3).

$$
P=\left\{\begin{array}{l}
2 \frac{A k T I}{q} \ln \left(\frac{I_{P H 1}-I / 3}{I_{0}}+1\right)-2 I^{2} R_{S} / 3- \\
\frac{n_{b} k T_{b} I}{q} \ln \left(\frac{I / 3-I_{P H 3}}{I_{o b}}+1\right), I_{P H 3}<I / 3<I_{P H 1} \\
2 \frac{A K T I}{q} \ln \left(\frac{I_{P H 1}-I / 3}{I_{0}}+1\right)-I^{2} R_{S}+ \\
\frac{A k T I}{q} \ln \left(\frac{I_{P H 3}-I / 3}{I_{0}}+1\right), 0<I / 3<I_{P H 3}
\end{array}\right.
$$

The corresponding I-U and P-U output characteristic curve can be draw according to the equation (2) and (3), as shown in Fig.3 and Fig.4 respectively. By observing Fig.3, the variation tendency of the curve shows that the current value is almost unchanged. And when the voltage increases to a certain value, the current begins to sharply reduce. It means the slope is zero at the very beginning and as the increasing of the voltage, the slope rapidly decreases.

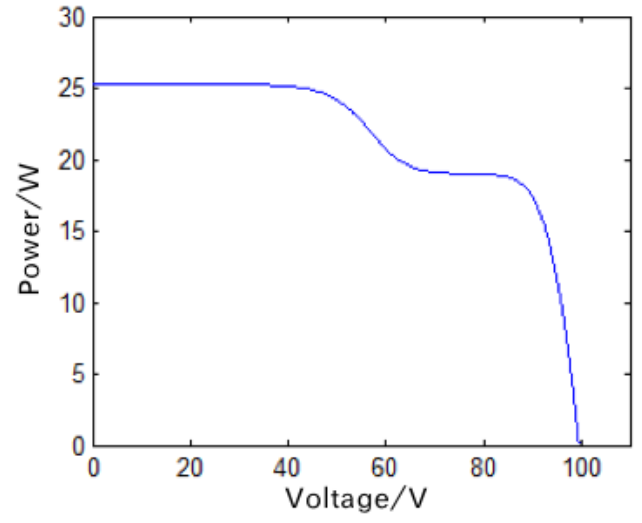

Fig.3 I-U characteristics of shaded array 


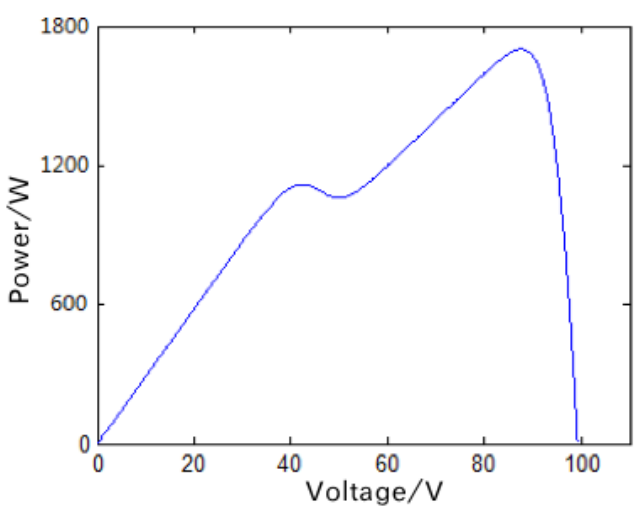

Fig.4 P-U characteristics of shaded array

By observing these characteristics, the maximum power point shows up only after the slope start to quickly decline. Then it is reasonable to initialize the particles of the PSO algorithm at a voltage value where the slope starts to quickly change. Here we choose the voltage at the point 'slope $=-3$ ' as the initial value of particles. Then instead of searching the whole voltage, the PSO algorithm just need to search the voltage from this initial value to the end. By doing so, it can helps narrow the searching area and track MPP faster.

\section{PSO-Based MPPT}

\subsection{General Overview of PSO}

Particle Swarm Optimization technique is an intelligent optimization technique developed by Dr. Eberhart and Dr. Kennedy[14], inspired by social behavior of bird foraging. The basic idea of Particle Swarm Optimization algorithm is through the cooperation and competition between particles to find the optimal solution.

PSO is initialized with a group of random particles and searches the best solution by updating generations. In each iteration, every particle is updated by private best value and global best value. Private best value is called $P_{\text {best }}$, which is the best solution (fitness) this particle has achieved so far. The global best value $\left(G_{\text {best }}\right)$ is the best solution (fitness) of the whole group. In each iteration, the particle knows where to go next by compare $P_{\text {best }}$ and $G_{\text {best }}$ and updates the velocity and the position by the following equations [15].

$$
\begin{gathered}
v_{i}^{k+1}=w v_{i}^{k}+c_{1} r_{1}\left(P_{\text {best }}-s_{i}^{k}\right)+c_{2} r_{2}\left(G_{\text {best }}-s_{i}^{k}\right) \\
s_{i}^{k+1}=s_{i}^{k}+v_{i}^{k+1}
\end{gathered}
$$

In the above equations, $\mathrm{i}$ is the number of particle, $\mathrm{k}$ is the itration time. $v_{i}^{k}$ is the velocity of ith particle within $\mathrm{k}$ iterations. $s_{i}^{k}$ is the position of ith particle within $\mathrm{k}$ iterations. The $\omega$ is used to keep the balance between global and private search. $c_{1}$ and $c_{2}$ are learning factors. $r_{1}$ and $r_{2}$ are two random numbers in $[-1,1]$.

\subsection{Improved PSO algorithm in MPPT}

As discussed before, due to the influence of partial shading and the mismatching of components, the output characteristics always tend to appear multi extreme situation. In this paper, we are focus on this situation and the others are similar. MPPT method control the output power of PV array by controlling the output voltage. The aim of MPPT method is to make sure the PV system works at a most efficient state.

PSO algorithm is one of the EA algorithms. It is widely used because of it's simple process, ease of implementation, and speed of calculation. Some researchers find that because of particle swarm optimization is based on search optimization, it must be capable of finding the maximum power point whatever the environment changes. The process of PSO algorithm, essentially, is an iterative process. In each iteration, follow the better fitness value, the particle moves toward to $G_{\text {best }}$. After several iterations, the velocity becomes very small or equals to zero, then the maximum power point is fund. In this paper, the objective function is defined as the measurement of PV array's output power and the position of the particle represents PV array's output voltage.

\subsubsection{Parameter setting and adjustment}

$\omega$ is inertia weight, which reflects the degree of inheritance of particle velocity. Smaller inertia weight makes the algorithm trapped in local extreme point and the larger inertia weight facilitate global search. But actually it is not easy to get the exact solution, therefore, to improve the efficiency of optimization, inertia weight is usually set from large to small. Generally $c_{1}$ is set from large to small and $c_{2}$ is set from small to large. So it is obviously that the adjustment of parameters plays a vital role in the optimization of PSO algorithm. In this paper, we set parameters: $\omega=1, c_{1}=c_{1}=1.49445$.

\subsubsection{Particle initialization}

General the choice of particle's initial value is arbitrary or according to the experience. The PSO methods in reference [8-10] select a number of points, which will reduce the optimization efficiency; The initializing of the particles in these methods is random, which is easy to cause reducing of optimization efficiency. As discussed above, to narrow the searching area, we choose the voltage at the point 'slope $=-3$ ' as the initial value of particles. By comparing to the traditional PSO algorithm, this method can track MPP faster.

\subsubsection{Terminate and restart conditions of PSO}

Similar to the traditional PSO algorithm, the proposed method also need to set up the terminate and restart conditions. The terminate condition is applied to avoid the oscillation near MPP. And the restart condition is used to restart PSO algorithm when the environment changed.

$$
\frac{\left|P\left(s_{i+1}\right)-P\left(s_{i}\right)\right|}{P\left(s_{i}\right)}>\Delta P
$$

In equation (6), $P\left(s_{i}\right)$ is the current maximum power. $P\left(s_{i+1}\right)$ is the output power of the next moment. $\Delta P$ is power variation. And we set $\Delta P=0.15$, which means PSO 
algorithm will be restarted when the power variation is greater than 0.15 .

\section{Simulation and Results}

\subsection{MATLAB simulation model Positioning}

MATLAB simulation model, as shown in Fig.5, is established in accordance with the contents mentioned above. To facilitate the analysis, simulation model of MPPT is established based on the BOOST circuit. The improved PSO algorithm is implemented by S-Function of MATLAB. In this study, we set DC bus voltage capacitanc $C_{2}=1000 \mu \mathrm{F}$. The inductance and capacitance of BOOST circuit are $\mathrm{L}=0.1 \mathrm{mH}$ and $C_{1}=1000 \mathrm{mH}$, The load resistance is set to $R=8.2 \Omega$.

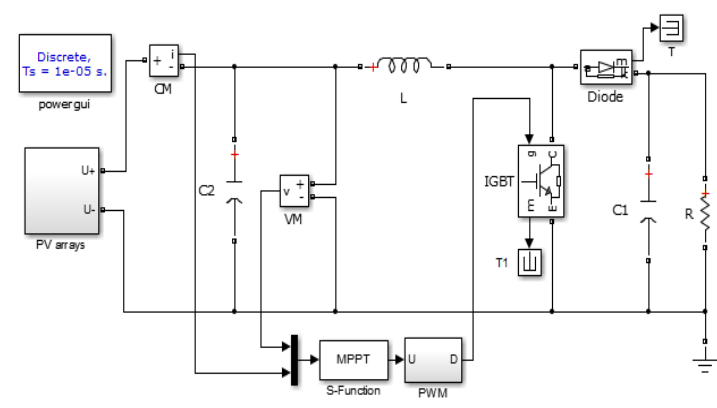

Fig.5 Simulation model of MPPT based on improved PSO

In this study, we set the output voltage of PV array to $U$ and the duty cycle of BOOST circuit to D. Load the booster of voltage U0 charged with constant value $=120$ $\mathrm{v}$, the calculation formula of duty cycle is as follow:

$$
D=1-U / U_{0}
$$

Before running the PSO algorithm, the BOOST circuit's duty cycle is set to 0 to get the open-circuit voltage, which is used to determine the bound of particle's position. In the optimization process, the positions of particles are updated by equation (4) and (5). Then the equation (7) is used to update duty cycle. Fitness values are updated by the output power of PV array. Finally, the method can make sure the PV system works at a most efficient state by optimizing the fitness value of each particles.

Under shading condition, the PV array's output characteristic are shown in Fig.3 and Fig.4. By observing the I-U characteristic, the PV system has two extreme value points. These two points are suitable as the initial value of the particles. From the I-P characteristic we can find that under this condition system does appear two extreme value point. One is local extreme value point $P_{1}=1098.4 \mathrm{~W}$ and another one is global extreme value point $P_{2}=1741.5 \mathrm{~W}$. Therefore, to improve the tracking efficiency of the PSO algorithm, it is reasonable to initialize the particles' position according to the I-U curve.

\subsection{Results}

Two versions of PSO are implemented in this study. The results are shown as below.

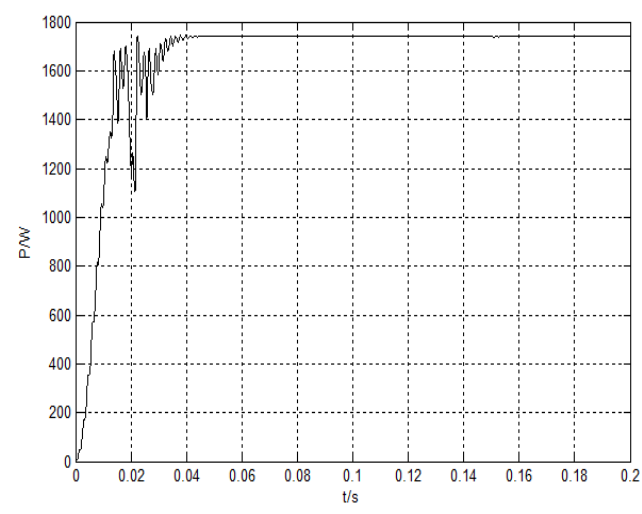

Fig.6 The tracking result of PSO under shading

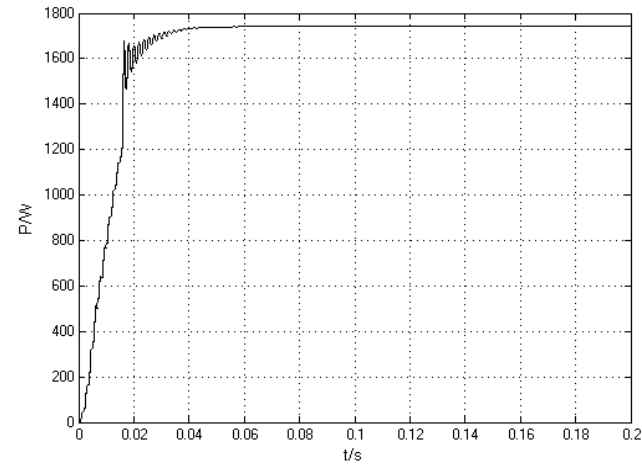

Fig.7 The tracking result of improved PSO under shading

Compared the basic PSO algorithm and improved PSO algorithm, we can find that under the condition of the partial shading both can accurate tracking to the MPP. Fig. 6 shows that when employing the basic PSO algorithm, we get large fluctuations in the optimization process as a result, which means it may cause misjudgment of MPP, and it took 0.044 seconds to find the MPP. The improved PSO algorithm shown in Fig. 7 shows a visibly smoother curve and took only 0.037 seconds to find the MPP, which is an improvement of 0.007 seconds. Therefore, this is definitive proof that the optimization makes the process more stable, and thus we can conclude that the improved PSO algorithm is more advantageous to the overall performance of the system.

\section{Conclusion}

In this paper, an improved PSO algorithm is used to track the MPP of a PV system. In order to improve the tracking speed, an initial value, determined by the analysing of the $\mathrm{I}-\mathrm{U}$ and $\mathrm{P}-\mathrm{U}$ characteristics curve, is given to the particles of PSO. In this proposed method, the fitness value is no longer determined by iteration and it is determined by the real-time sampling of output power. It is shown that the proposed method can track MPP faster and has less fluctuation. The efficiency and practicability of the algorithm has been improved.

\section{Acknowledgement}


The authors would like to thank the Natural Science Foundation of Zhejiang, China (Grant No. LYE0700006).

\section{Reference}

1. A Chen, L Feng, C S Du, C H Zhang, Modeling of Photovoltaic Array Based on Support Vector Machines Under Partial Shaded Conditions, Transactions of China Electrotechnical Society, Vol.26, No.3 (2011)

2. Y H Yang, K L Zhou, Photovoltaic Cell Modeling and MPPT Control Strategies, Transactions of China Electrotechnical Society, Vol.26, Sup.1 (2011)

3. M Dong, J Yang, K Peng, An Luo, Zero Average Incremental Conductance Maximum Power Point Tracking Control for Photovoltaic System, Proceedings of the CSEE, Vol.30, No.21 (2010)

4. Parlak K S, Can H. A new MPPT method for PV array system under partially shaded conditions[C]. IEEE International Symposium on Power Electronics for Distributed Generation Systems (PEDG), 437-441 (2012)

5. X Zhang, R X Cao, Solar Photovoltaic(PV) Grid Generation and Its Inverter Control[M], Mechanical Industry Press (2011)

6. Ramaprabha R, Mathur B, Ravi A, et al. Modified Fibonacci search based MPPT scheme for SPVA under partial shaded conditions[C]//3rd International Conference on Emerging Trends in Engineering and Technology. Goa, India: IEEE, 379-384 (2010)

7. Z Cheng, $\mathrm{H}$ Zhou, $\mathrm{H} Z$ Y Yang. Research on MPPT control of PV system based on PSO algorithm[J]. Control and Decision Conference (CCDC), Chinese, 887-892 (2010)

8. Y W Zhu, X C Shi, Y Q Dan, P Li, W Y Liu, D B Wei, C Fu, Application of PSO Algorithm in Global MPPT for PV Array, Proceedings of the CSEE, Vol.32, No.4 (2012)

9. Miyatake M, Veerachary M, Toriumi F. Maximum power point tracking of multiple photovoltaic arrays : a PSO approach[J], IEEE Transactions on Aerospace and Electronic Systems, 47(01) :367-380 (2011)

10. Phinmasone V, Kondo $\mathrm{Y}$, Kamejima $\mathrm{T}$, et al. Evaluation of Extracted Energy from PV with PSObased MPPT against Various Types of Solar Irradiation Changes[C]. International Conference on Electrical Machines and Systems (ICEMS). IEEE, 487-492 (2010)

11. H H Bian, Q S Xu, S Gao, Kazuto Yukita, Katsuhiro Ichiyanagi, Operation Mismatches of Photovoltaic Array Considering Random Shadows, Transactions of China Electrotechnical Society, Vol.25, No.6 (2010)

12. L Tang, C B Zeng, H Miao, W Xu, Y H Zhang, Y Y Liu, A Novel Maximum Power Point Tracking Scheme for PV Systems Under Partially shaded Conditions Based on Monte Carlo Algorithm,
Transactions of China Electrotechnical Society, Vol.30, No.1 (2015)

13. Z T Zhai, X F Cheng, J L Ding, J Zha, Characteristics of partially shadowed serial PV module ouput, Journal of Unversity of Science and Technology of China, Vol.39, No.4 (2009)

14. Khanesar M A, Teshnehlab M, Shoorehdeli M A. A novel binary particle swarm optimization[C], Proceedings of IEEE Conference on Control\&Automation, Mediterranean IEEE Control Systems Society, 1-6 (2007)

15. Phinmasone V, Kondo Y,Shiota N, et al. The Demonstration Experiments to Verify the Effectiveness of the Improved PSO-based MPPT Controlling Multiple Photovoltaic Arrays[C].ECCE Asia Downunder(ECCE Asia).IEEE, 86-92 (2013) 